\title{
Diretrizes para o Projeto do Algoritmo LMS Aplicado à Super-Resolução de Vídeo
}

\author{
Guilherme Holsbach Costa ${ }^{\dagger}$ e José Carlos Moreira Bermudez*
}

\begin{abstract}
Resumo-A reconstrução com super-resolução (SRR) de imagens consiste basicamente em combinar diversas imagens de baixa-resolução de uma mesma cena para formar uma imagem com resolução mais alta. As principais características dessa técnica são a elevada sensibilidade a erros de registro, a dificuldade de modelagem dos sinais de imagem (outliers) e o elevado custo computacional. Este trabalho avança no projeto do algoritmo LMS aplicado à SRR. Baseado em uma análise estatística, são propostas diretrizes específicas que levam a um desempenho próximo do ótimo, quando situações práticas de aplicação são consideradas. A análise também mostra que, diferente do comportamento do LMS tradicional, pequenos passos de adaptação não levam necessariamente a erros quadráticos médios menores em regime permanente.
\end{abstract}

Palavras-Chave-Reconstrução de imagens, registro de imagens, LMS, estimação adaptativa.

Abstract-The super-resolution image reconstruction (SRR) consists basically of combining multiple low resolution images of the same scene to form a higher resolution image. The main characteristics of this technique are the high sensitivity to the registration errors, the difficulty in modeling the image signals (outliers) and the high computational cost. This work advances in the design of the LMS algorithm applied to SRR. Based on a statistical analysis, we propose design guidelines that lead to a close-to-optimum algorithm performance when considering practical situations. The analysis also shows that, differently from the traditional LMS behavior, lower step-size values do not necessarily lead to better steady-state mean-square errors.

Keywords-Image reconstruction, image registration, LMS, adaptive estimation.

\section{INTRODUÇÃO}

A reconstrução com super-resolução (SRR - superresolution reconstruction) consiste basicamente em combinar múltiplas imagens de baixa resolução para formar uma imagem de alta (maior) resolução. Em [1] são revisados importantes resultados em SRR disponíveis na literatura.

Um dos maiores problemas em SRR é a sensibilidade dos algoritmos a erros de modelagem [2]. Outliers são definidos como dados cuja distribuição não segue a assumida na modelagem do problema. No contexto do movimento, outliers são freqüentemente regiões da cena que sofrem oclusão ou que são descobertas repentinamente em uma seqüência de imagens, i.e., são as inovações que ocorrem entre dois quadros consecutivos. Vários trabalhos têm abordado o problema dos outliers em SRR [2], [3], [4]. Em [3] é proposto um algoritmo robusto aos outliers causados por erros de registro, de

$\dagger^{*}$ Departamento de Engenharia Elétrica, Universidade Federal de Santa Catarina, Florianópolis, Brasil. ${ }^{\dagger} \mathrm{O}$ autor está atualmente com a Universidade de Caxias do Sul. E-mails: holsbach@gmail.com, j.bermudez@ieee.org. Este trabalho foi parcialmente financiado pelo CNPq (141456/2003-5 e 308095/2003-0). modelagem do sistema óptico (blurring), e de modelagem do ruído, assim como aos outliers causados pelo deslocamento de objetos (inovações) na cena. Nesse algoritmo, o estimador de média, tradicionalmente usado para calcular o gradiente em algoritmos de SRR, é substituído por um estimador de mediana, mais robusto aos outliers. Em [2], [4], o efeito dos outliers na SRR é discutido e um algoritmo robusto é proposto, baseado na norma $L_{1}$.

Quando a aplicação de interesse requer processamento em tempo-real, a complexidade computacional precisa ser considerada. Em [5], é proposto um algoritmo adaptativo aplicado à SRR de uma sequiência de imagens, com grande vantagem computacional em relação aos demais existentes. $\mathrm{O}$ algoritmo segue a estimação least-mean-squares tradicional, e, de forma a diferenciar-se do algoritmo LMS tradicional, é chamado LMS-SRR. Mais recentemente, outro algoritmo robusto aos outliers foi proposto [4], o qual admite uma implementação rápida para os casos em que o movimento relativo entre as imagens poder ser considerado translacional e global. Entretanto, mesmo sob tais condições, seu custo computacional e requisitos de memória não são competitivos com o LMS-SRR.

O projeto do algoritmo LMS-SRR é estudado em [6]. Baseado no momento de segunda ordem do erro de reconstrução, orientações de projeto foram fornecidas com o intuito de reduzir o efeito dos outliers na seqüência de imagens reconstruídas. A análise estatística em [6] considera outliers originados a partir de inovações entre as imagens da seqüência, e negligencia o efeito dos erros de registro.

Neste trabalho, a estratégia para a escolha dos parâmetros do algoritmo LMS-SRR proposta em [6] é aperfeiçoada, levando em conta também os efeitos dos erros de registro. Diretrizes específicas são propostas para a escolha do passo de adaptação e do número de iterações a serem realizadas pelo algoritmo para cada amostra do sinal de entrada, de forma a minimizar o efeito dos outliers na seqüência de imagens reconstruídas. $\mathrm{O}$ efeito desses dois parâmetros no desempenho do algoritmo é estudado, e é mostrado que, diferente do comportamento do algoritmo LMS tradicional, a redução do passo de convergência nem sempre leva a uma redução na velocidade do LMS-SRR ou a melhores estimações em regime permanente.

Na Seção II, é apresentada uma breve revisão do algoritmo LMS-SRR [5]. Na Seção III, é apresentada a análise do algoritmo. Na Seção IV, é proposta a estratégia de projeto visando a robustez aos outliers. Na Seção V, são apresentados resultados de simulações que ilustram a validade e benefícios da estratégia de projeto proposta. Finalmente, na Seção VI, este trabalho é concluído. 


\section{O ALGORITMO LMS-SRR}

\section{A. Modelagem dos sinais}

Dada a representação matricial $N \times N$ de uma imagem digital de baixa-resolução (observada) $\mathbf{Y}(t)$ e a representação matricial $M \times M(M>N)$ de uma imagem original, de altaresolução, $\mathbf{X}(t)$, o processo de aquisição pode ser modelado como [1]

$$
\mathbf{y}(t)=\mathbf{D}(t) \mathbf{x}(t)+\mathbf{e}(t),
$$

em que os vetores $\mathbf{y}(t)\left(N^{2} \times 1\right)$ e $\mathbf{x}(t)\left(M^{2} \times 1\right)$ são respectivamente as representações lexicográficas das imagens observada e original, no instante de tempo (discreto) $t$. A matriz $\mathbf{D}(t)$ $\left(N^{2} \times M^{2}\right)$ modela a degradação devida à subamostragem e distorções ópticas (blurring), e é assumida conhecida. $\mathrm{O}$ vetor $\mathbf{e}(t)\left(N^{2} \times 1\right)$ modela o ruído de observação (eletrônico), assumido espacialmente estacionário, estatisticamente independente de $\mathbf{y}(t)$ e $\mathbf{x}(t)$, branco, gaussiano, com média zero e com matriz de autocorrelação espacial $\mathbf{R}_{\mathbf{e}}(t)=\sigma_{e}^{2}(t) \mathbf{I} \cdot \sigma_{e}^{2}(t)$ é assumida ser determinada a partir de testes com o sensor de aquisição.

A dinâmica do sinal de entrada é modelada por [5]

$$
\mathbf{x}(t)=\mathbf{G}(t) \mathbf{x}(t-1)+\mathbf{s}(t)
$$

em que a matriz de registro $\mathbf{G}(t)$ descreve o deslocamento relativo dos pixels de $\mathbf{x}(t-1)$ para $\mathbf{x}(t)$. $O$ vetor $\mathbf{s}(t)$ modela as inovações em $\mathbf{x}(t)$, e portanto inclui na imagem reconstruída as contribuições dos outliers causados pelo movimento.

\section{B. O algoritmo adaptativo LMS-SRR}

O algoritmo LMS-SRR busca minimizar o erro quadrático médio (MSE - mean-square error) $\mathrm{E}\left\{\|\mathbf{y}(t)-\mathbf{D}(t) \hat{\mathbf{x}}(t)\|^{2}\right\}$ [5], em que $\mathrm{E}\{\cdot\}$ denota esperança estatística. Portanto, a função custo é definida como $\mathbf{J}_{\mathrm{MS}}(t)=\mathrm{E}\left\{\|\boldsymbol{\epsilon}(t)\|^{2} \mid \hat{\mathbf{x}}(t)\right\}$. Seguindo o método Steepest Descent, $\hat{\mathbf{x}}(t)$ é atualizado na direção negativa do gradiente. Note que a superfície de desempenho $\mathbf{J}_{\mathrm{MS}}(t)$ é definida para um instante de tempo específico $t$.

O algoritmo LMS-SRR é a versão estocástica do algoritmo Steepest Descent. Usando a estimativa instantânea de $\nabla \mathbf{J}_{\mathrm{MS}}(t)$, tem-se

$$
\hat{\mathbf{x}}_{k+1}(t)=\hat{\mathbf{x}}_{k}(t)+\mu \mathbf{D}^{\mathrm{T}}(t)\left[\mathbf{y}(t)-\mathbf{D}(t) \hat{\mathbf{x}}_{k}(t)\right],
$$

que corresponde à equação de atualização do LMS-SRR. A atualização temporal de (3) dá-se baseada na dinâmica do sinal (2), e é determinada pela matriz de registro $\mathbf{G}(t)$ :

$$
\hat{\mathbf{x}}_{0}(t+1)=\mathbf{G}(t+1) \hat{\mathbf{x}}_{K}(t),
$$

em que $K$ é o número de iterações desempenhadas pelo LMSSRR para cada amostra temporal de entrada.

\section{ANÁLISE}

Deste ponto em diante, $\mathbf{G}(t)$ é assumida conhecida (determinística) e representa a matriz de movimento real (livre de erros de estimação de movimento). A matriz $\hat{\mathbf{G}}(t)$ representa a matriz de registro estimada (com erros).
Uma vez que apenas a estimativa $\hat{\mathbf{G}}(t)$ está disponível durante a reconstrução, as equações de atualização do LMSSRR para esse caso devem ser definidas por (3) e por:

$$
\hat{\mathbf{x}}_{0}(t+1)=\hat{\mathbf{G}}(t+1) \hat{\mathbf{x}}_{K}(t),
$$

com $\hat{\mathbf{G}}(t)$ modelada como [5], [7]

$$
\hat{\mathbf{G}}(t)=\mathbf{G}(t)+\Delta \mathbf{G}(t),
$$

em que $\boldsymbol{\Delta} \mathbf{G}(t)$ é uma matriz aleatória, definida pelas características do método de registro e da sequiência de imagens. Agrupando-se (3) e (4), chega-se em uma única equação de atualização, recursiva, para o algoritmo LMS-SRR com $K$ iterações por amostra de entrada:

$$
\hat{\mathbf{x}}(t)=\mathbf{A}^{K}(t) \hat{\mathbf{G}}(t) \hat{\mathbf{x}}(t-1)+\mu \sum_{n=0}^{K-1} \mathbf{A}^{n}(t) \mathbf{D}^{\mathrm{T}}(t) \mathbf{y}(t),
$$

em que $\mathbf{A}(t)=\left[\mathbf{I}-\mu \mathbf{D}^{\mathrm{T}}(t) \mathbf{D}(t)\right]$

Definindo o erro de reconstrução de imagens (em altaresolução) como $\mathbf{v}(t)=\hat{\mathbf{x}}(t)-\mathbf{x}(t)$ e usando (3), (4), (6) e (7), chega-se em [7]:

$$
\begin{aligned}
\mathbf{v}(t)= & \mathbf{A}^{K}(t) \mathbf{G}(t) \mathbf{v}(t-1)+\mathbf{A}^{K}(t) \boldsymbol{\Delta} \mathbf{G}(t) \hat{\mathbf{x}}(t-1) \\
& -\mathbf{A}^{K}(t) \mathbf{s}(t)+\mu \sum_{n=0}^{K-1} \mathbf{A}^{n}(t) \mathbf{D}^{\mathrm{T}}(t) \mathbf{e}(t) .
\end{aligned}
$$

A Eq. (8) pode ser usada para determinar o comportamento das flutuações do erro de reconstrução.

\section{A. Hipóteses estatísticas e aproximações}

$\mathrm{O}$ estudo das propriedades estatísticas de $\mathbf{v}(t)$ requer simplificações, de forma a tornar o problema matematicamente tratável. As seguintes aproximações e hipóteses são usadas na análise que se segue: (A1) As inovações $\mathbf{s}(t)$ são assumidas com média zero, com matriz de autocorrelação $\mathbf{R}_{\mathbf{s}}(t)\left(M^{2} \times M^{2}\right)$, e descorrelacionadas de $\mathbf{x}(t-1)$. Esta mesma hipótese é usada em [8]. (A1) é válida, por exemplo, quando outliers estão presentes devido ao movimento entre quadros e a condição de fronteira de Neumann é assumida na construção de $\mathbf{G}(t)$. Esse é um caso importante, uma vez que a condição de Neumann provê os resultados de reconstrução com a melhor qualidade perceptual [9]. Esta hipótese não será válida quando a condição de Dirichlet (zero-padding) for assumida [10], uma vez que o preenchimento com zeros faz com que $\mathbf{s}(t)$ sempre assuma valores não-negativos $\mathrm{e}$ correlacionados com $\mathbf{x}(t-1)$. (A2) As inovações $\mathbf{s}(t)$ são assumidas estatisticamente independentes dos erros de registro $\Delta \mathbf{G}(t)$. (A3) O vetor de ruído de observação e $(t)$ é assumido estatisticamente independente dos erros de registro $\Delta \mathbf{G}(t)$ e de qualquer outro sinal no sistema.

\section{B. Comportamento do erro quadrático médio de reconstrução}

Para estudar o desempenho do algoritmo, o erro quadrático médio de reconstrução (MSRE - mean-square reconstruction error) é definido como MSRE $=\mathrm{E}\left[\mathbf{v}^{\mathrm{T}}(t) \mathbf{v}(t)\right] / M^{2}$, em que $M^{2}$ é o número de pixels das imagens de alta-resolução. Essa norma pode ser avaliada via $\operatorname{tr}\{\mathbf{K}(t)\}=\operatorname{tr}\left\{\mathrm{E}\left[\mathbf{v}(t) \mathbf{v}^{\mathrm{T}}(t)\right]\right\}$, 
em que $\mathbf{K}(t)$ é a matriz de autocorrelação de $\mathbf{v}(t)$, e $\operatorname{tr}\{\cdot\}$ denota o traço de uma matriz.

Pós-multiplicando-se (8) pela sua transposta, tirando-se o valor esperado, e usando-se $A 1, A 2$ e $A 3$, tem-se

$$
\begin{aligned}
\mathbf{K}(t) & =\mathbf{A}^{K}(t)\{\mathrm{E}\{[\mathbf{G}(t) \mathbf{v}(t-1)+\Delta \mathbf{G}(t) \hat{\mathbf{x}}(t-1)] \\
& \left.\left.\times[\mathbf{G}(t) \mathbf{v}(t-1)+\Delta \mathbf{G}(t) \hat{\mathbf{x}}(t-1)]^{\mathrm{T}}\right\}+\mathbf{R}_{\mathbf{s}}(t)\right\} \mathbf{A}^{K}(t) \\
& +\mu^{2} \sum_{n=0}^{K-1} \mathbf{A}^{n}(t) \mathbf{D}^{\mathrm{T}}(t) \mathbf{R}_{\mathbf{e}} \mathbf{D}(t) \sum_{m=0}^{K-1} \mathbf{A}^{m}(t) .
\end{aligned}
$$

A matriz simétrica $\mathbf{A}(t)$ pode ser decomposta como

$$
\mathbf{A}(t)=\mathbf{Q}(t) \boldsymbol{\Gamma}(t) \mathbf{Q}^{\mathrm{T}}(t),
$$

em que $\mathbf{Q}(t)$ é a matriz (ortogonal) de autovetores de $\mathbf{A}(t)$ e $\boldsymbol{\Gamma}(t)$ é a matriz (real e diagonal) de autovalores de $\mathbf{A}(t)$. Além disso, $\mathbf{D}^{\mathrm{T}}(t) \mathbf{D}(t)$ tem os mesmos autovetores de $\mathbf{A}(t)$, e $\mathbf{D}^{\mathrm{T}}(t) \mathbf{D}(t)=\mathbf{Q}(t) \boldsymbol{\Lambda}(t) \mathbf{Q}^{\mathrm{T}}(t)$. Usando-se esta última igualdade e $\mathbf{A}(t)=\left[\mathbf{I}-\mu \mathbf{D}^{\mathrm{T}}(t) \mathbf{D}(t)\right]$ em (10), segue que

$$
\boldsymbol{\Gamma}(t)=[\mathbf{I}-\mu \boldsymbol{\Lambda}(t)] .
$$

Considerando (9), (10) e (11), e usando a propriedade comutativa do traço do produto de matrizes, chega-se no seguinte modelo para o comportamento do MSRE:

$$
\begin{aligned}
\operatorname{tr}[\mathbf{K}(t)] & =\operatorname{tr}\left\{\boldsymbol{\Gamma}^{2 K}(t) \mathbf{Q}^{\mathrm{T}}(t) \mathbf{R}_{\mathbf{s}}(t) \mathbf{Q}(t)\right. \\
& +\mu^{2} \sum_{m=0}^{K-1} \boldsymbol{\Gamma}^{m}(t) \sum_{n=0}^{K-1} \boldsymbol{\Gamma}^{n}(t) \mathbf{Q}^{\mathrm{T}}(t) \mathbf{D}^{\mathrm{T}}(t) \mathbf{R}_{\mathbf{e}} \mathbf{D}(t) \mathbf{Q}(t) \\
& +\boldsymbol{\Gamma}^{2 K}(t) \mathbf{Q}^{\mathrm{T}}(t) \mathrm{E}\{[\mathbf{G}(t) \mathbf{v}(t-1)+\boldsymbol{\Delta} \mathbf{G}(t) \hat{\mathbf{x}}(t-1)] \\
& \left.\left.\times[\mathbf{G}(t) \mathbf{v}(t-1)+\boldsymbol{\Delta} \mathbf{G}(t) \hat{\mathbf{x}}(t-1)]^{\mathrm{T}}\right\} \mathbf{Q}(t)\right\}
\end{aligned}
$$

\section{ESCOLHA DOS PARÂMETROS DO LMS-SRR}

A Equação (12) pode ser reescrita como

$$
\operatorname{tr}[\mathbf{K}(t)]=\operatorname{tr}\left\{\boldsymbol{\Gamma}^{2 K}(t) \mathbf{M}_{1}(t)+\boldsymbol{\Gamma}^{2 K}(t) \mathbf{M}_{2}(t)+\mathbf{T}[\mathbf{e}(t)]\right\},
$$

em que

$$
\begin{aligned}
\mathbf{M}_{1}(t)= & \mathbf{Q}^{\mathrm{T}}(t) \mathrm{E}\{[\mathbf{G}(t) \mathbf{v}(t-1)+\Delta \mathbf{G}(t) \hat{\mathbf{x}}(t-1)] \\
& \left.\times[\mathbf{G}(t) \mathbf{v}(t-1)+\Delta \mathbf{G}(t) \hat{\mathbf{x}}(t-1)]^{\mathrm{T}}\right\} \mathbf{Q}(t) \\
\mathbf{M}_{2}(t)= & \mathbf{Q}^{\mathrm{T}}(t) \mathbf{R}_{\mathbf{s}}(t) \mathbf{Q}(t) \\
\mathbf{T}[\mathbf{e}(t)]= & \mu^{2} \sum_{m=0}^{K-1} \boldsymbol{\Gamma}^{m}(t) \sum_{n=0}^{K-1} \boldsymbol{\Gamma}^{n}(t) \mathbf{Q}^{\mathrm{T}}(t) \mathbf{D}^{\mathrm{T}}(t) \mathbf{R}_{\mathbf{e}} \mathbf{D}(t) \mathbf{Q}(t) .
\end{aligned}
$$

Em (13), $\mathbf{M}_{1}(t)$ é o termo responsável por introduzir os efeitos dos erros de registro, enquanto $\mathbf{M}_{2}(t)$ introduz o efeito das inovações. Além disso, $\mathbf{M}_{1}(t)$ afeta o comportamento do MSRE em regime transitório de $\operatorname{tr}[\mathbf{K}(t)]$, uma vez que depende de $\mathbf{v}(t-1)$. $\mathbf{T}[\mathbf{e}(t)]$ é a parcela referente à contribuição do ruído de observação e $(t)$.

A análise a seguir concentra-se na redução dos dois primeiros termos de (13), uma vez que estes são responsáveis pelas influências mais significativas em aplicações práticas ${ }^{1}$, e porque a indisponibilidade de informação suficiente sobre a relação entre $\mathbf{M}_{1}(t), \mathbf{M}_{2}(t)$ e $\mathbf{R}_{\mathbf{e}}$ impossibilita o uso de $\mathbf{T}[\mathbf{e}(t)]$ em uma estratégia de otimização.

A fim de, inicialmente, reduzir a influência das inovações no resultado de reconstrução, uma possível estratégia é escolher valores para $\mu$ e $K$ de forma a reduzir o efeito sobre o traço de $\mathbf{K}(t)$ do termo em (13) envolvendo $\mathbf{M}_{2}(t)$. Como será mostrado, $\mathbf{M}_{1}(t)$ e $\mathbf{M}_{2}(t)$ possuem propriedades similares. Assim, a estratégia proposta também reduzirá o efeito do termo envolvendo $\mathbf{M}_{1}(t)$, o qual afeta tanto a taxa de convergência quanto o efeito dos erros de registro no desempenho do algoritmo. Uma vez que as matrizes $\mathbf{M}_{1}(t)$ e $\mathbf{M}_{2}(t)$ em (14) e (15), respectivamente, não podem ser diretamente controladas por $\mu$ ou $K$, tal estratégia concentra-se em reduzir os dois primeiros termos em (13) pelo ajuste de $\Gamma^{2 K}(t)$.

As seguintes propriedades são importantes para a estratégia de projeto a ser proposta na próxima seção: (a) Ambas matrizes $\mathbf{M}_{1}(t)$ e $\mathbf{M}_{2}(t)$ ( $\mathbf{R}_{\mathbf{s}}$ é semi-definida positiva) podem ser decompostas como produtos de matrizes reais por suas transpostas. Portanto, ambas são matrizes semi-definidas positivas [11, p.558]; (b) Sendo $\Gamma^{2 K}(t)$ uma matriz diagonal, seus elementos atuam como ponderadores das linhas de $\mathbf{M}_{1}(t)$ e $\mathbf{M}_{2}(t)$ em (14); (c) Os elementos de $\boldsymbol{\Gamma}^{2 K}(t)$ são não-negativos para quaisquer valores de $K$ e $\mu$; $(d)$ Uma vez que $\mathbf{M}_{1}(t)$ e $\mathbf{M}_{2}(t)$ são semi-definidas positivas, os elementos de suas diagonais são não-negativos.

\section{A. Escolha de $\mu$ e $K$}

Em aplicações práticas, informações precisas sobre os elementos de $\mathbf{M}_{1}(t)$ e $\mathbf{M}_{2}(t)$ não estão disponíveis. Entretanto, é razoável assumir o conhecimento de $\mathbf{D}(t)$, que pode ser obtido uma vez que se conheça detalhes sobra a implementação do sistema. Assim, $\mu$ e $K$ podem ser escolhidos de forma a minimizar os autovalores de $\mathbf{A}^{2 K}(t)$ (que são elementos de $\left.\boldsymbol{\Gamma}^{2 K}(t)\right)$ em algum sentido, dado que se conheça $\mathbf{D}(t)$ e respeitando o limite de estabilidade do algoritmo [5].

Desprezando o último termo, $\mathbf{T}[\mathbf{e}(t)]$, a Eq. (13) pode ser reescrita como

$$
\operatorname{tr}[\mathbf{K}(t)] \simeq \sum_{i=1}^{M^{2}}\left[1-\mu \lambda_{i}(t)\right]^{2 K}\left[m_{2_{i, i}}(t)+m_{1_{i, i}}(t)\right],
$$

em que $M^{2}$ é o número de pixels das imagens de altaresolução, e $m_{1_{i, i}}(t)$ e $m_{2_{i, i}}(t)$ são, respectivamente, os $(i, i)$ ésimos elementos de $\mathbf{M}_{1}(t)$ e $\mathbf{M}_{2}(t)$. Uma vez que nenhuma informação precisa sobre esses elementos pode ser obtida $a$ priori (sabe-se apenas que eles são não-negativos), resta a possibilidade de reduzir $\operatorname{tr}[\mathbf{K}(t)]$ por meio da minimização do máximo valor de $\left[1-\mu \lambda_{i}(t)\right]^{2 K}$.

$\mathrm{O}$ passo de adaptação do LMS-SRR deve ser não-negativo e seu limite de estabilidade é dado por [5]

$$
\mu<\frac{2}{\lambda_{\max }},
$$

\footnotetext{
${ }^{1}$ A influência do ruído de observação e $(t)$ pode ser desprezada quando comparada a outras fontes de erro como, por exemplo, registro inacurado e problemas de modelagem das inovações, na maioria das aplicações práticas [1], [7].
} 


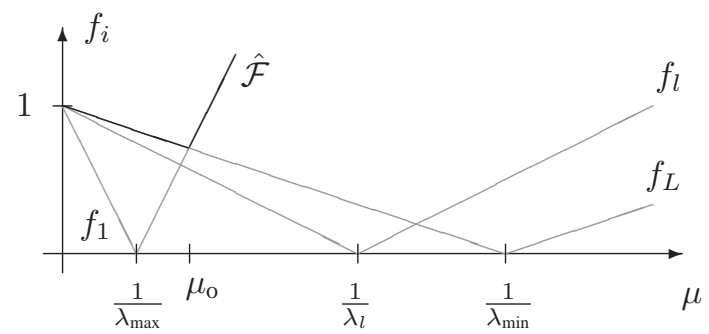

Fig. 1. Problema Minimax.

em que $\lambda_{\max }$ é o máximo autovalor de $\mathbf{D}^{\mathrm{T}}(t) \mathbf{D}(t)$. Portanto, é fácil mostrar que $\left[1-\mu \lambda_{i}(t)\right]^{2}<1$ para todos os valores válidos de $\mu$ e para todos os valores de $i$ e $t$. Assim, $\lim _{K \rightarrow \infty}\left[1-\mu \lambda_{i}(t)\right]^{2 K} \rightarrow 0$ para todo $\lambda_{i}(t) \neq 0$. Então, exceto sob condições especiais (ideais) [6], a escolha natural de $K$ é o maior número possível de iterações, limitado apenas pelo custo computacional, pelo hardware disponível, e por outros possíveis requisitos específicos da aplicação tempo-real em questão.

Assumindo um valor pré-determinado (fixo) para $K$, a escolha do passo de convergência $\mu$ pode ser formulada como o problema de otimização minimax

$$
\mu_{\mathrm{o}}=\min _{\mu}\{\mathcal{F}\} \text {, em que } \mathcal{F}=\max _{i} f_{i}^{2 K} \text {, sujeito a }\left|f_{i}\right|<1,
$$

com $f_{i}=1-\mu \lambda_{i}(t)$. Este problema pode ser simplificado conforme:

$$
\mu_{\mathrm{o}}=\min _{\mu}\{\hat{\mathcal{F}}\} \text {, em que } \hat{\mathcal{F}}=\max _{i}\left|f_{i}\right| \text {, sujeito a }\left|f_{i}\right|<1 \text {. }
$$

Deste ponto em diante, apenas os valores positivos de $\lambda_{i}(t)$ são considerados. Uma vez que $f_{i}$ não é função de $\mu$ para $\lambda_{i}(t)=0$, não faz sentido que esses valores sejam incluídos na minimização. Em implementações reais, podem ser considerados no problema de otimização apenas os autovalores mais significativos.

Na Figura 1 é mostrado valor absoluto dos valores de $f_{i}$ e $\mathcal{F}$, assumindo que $\lambda_{1}(t)>\lambda_{l}(t)>\lambda_{L}(t)$ [12]. O mínimo de $\hat{\mathcal{F}}$ ocorre para

$$
-\left[1-\mu \lambda_{\max }(t)\right]=\left[1-\mu \lambda_{\min }(t)\right] .
$$

Resolvendo-se (21) para $\mu$ tem-se que

$$
\mu_{\mathrm{o}}=\frac{2}{\lambda_{\max }(t)+\lambda_{\min }(t)} .
$$

$\mathrm{O}$ ponto $\mu=\mu_{\mathrm{o}}$ leva à $\left[1-\mu_{\mathrm{o}} \lambda_{\max }(t)\right]^{2 K}=\left[1-\mu_{\mathrm{o}} \lambda_{\min }(t)\right]^{2 K}$

\section{Resultados}

\section{A. Condições gerais de simulação}

Quando o algoritmo LMS é aplicado na SRR, uma boa inicialização pode ser obtida a partir da interpolação bicúbica da imagem observada no instante inicial. Entretanto, nos exemplos a seguir o algoritmo é inicializado longe do ótimo, de forma que se possa avaliar o seu comportamento durante o regime transitório de adaptação, e não apenas durante o regime permanente. Como este fim, o algoritmo LMS-SRR é inicializado em todas as simulações com imagens sintéticas construídas a partir de uma única realização de um processo $\operatorname{WGN}(0,1)$, com as amplitudes dos pixels normalizadas para se adequarem à escala dinâmica das imagens [0, 255].

A matriz $\mathbf{D}(t)$ é assumida estacionária $(\mathbf{D}(t)=\mathbf{D})$. As distorções ópticas (blurring) foram implementadas através de uma filtragem espacial com máscara gaussiana de tamanho $6 \times 6$ e variância 1.0 (desempenhada sobre uma subamostragem impulsiva $)^{2}$. Neste caso, os $\lambda_{i}$ assumem diversos valores distintos. Para a determinação do passo de convergência ótimo, é considerado o conjunto dos maiores autovalores de $\mathbf{D}^{\mathrm{T}} \mathbf{D}$ tal que a soma de seus elementos seja igual ou maior do que noventa por cento da soma de todos os autovalores. Os maiores autovalores variam de $\lambda_{\min }=0.0422$ a $\lambda_{\max }=0.25$. De acordo com (18), o máximo passo de adaptação é $\mu_{\max }=8$, e de (22) o passo proposto é $\mu_{\mathrm{o}} \simeq 6,8$.

Exceto quando devidamente especificadas, são consideradas imagens de alta-resolução com $64 \times 64$ pixels, imagens de baixa-resolução com $32 \times 32$ pixels, e apenas uma iteração do algoritmo por amostra temporal do sinal de entrada $(K=1)$. Para construir a matriz de registro $\mathbf{G}(t)$ e a sua estimativa $\hat{\mathbf{G}}(t)$, a condição de fronteira de Neumann é utilizada. Tal opção é adotada por ser esta a condição mais empregada em situações práticas.

Simulações de Monte Carlo (MC) consistem na média de 100 realizações, considerando diferentes seqüências de imagens. Nesses casos, as seqüências foram geradas sinteticamente, a partir de imagens naturais e de vetores de movimentos translacionais e globais, conforme [7].

\section{B. Exemplo 1: Robustez aos outliers}

Neste exemplo, os vetores de deslocamento global usados para gerar as seqüências são assumidos conhecidos para cada instante de tempo $t$ (erros de registro não são considerados). $\mathrm{O}$ ruído aditivo é considerado nulo $\left(\sigma_{e}^{2}(t)=0\right)$. Essas duas condições têm o objetivo de isolar os efeitos dos outliers. Os vetores de deslocamento global foram gerados a partir de incrementos unitários em ambas as direções, horizontal e vertical (simulando um movimento diagonal da câmera), na grade de alta-resolução, a cada instante de tempo $t$.

Na Figura 2 são mostrados os resultados obtidos para diferentes valores de $\mu$. Note que embora $\mu_{\mathrm{o}}$ não corresponda ao valor ótimo de $\mu,{ }^{3}$ ele leva a um desempenho do algoritmo bastante próximo ao desempenho ótimo.

\section{Exemplo 2: Robustez aos erros de registro}

Neste exemplo, os vetores de deslocamento global utilizados para gerar as sequiências de alta-resolução foram gerados a partir de um processo random walk. Os vetores de deslocamento foram, depois de gerados, estimados através do algoritmo de registro proposto em [13]. Cabe ressaltar, entretanto, que a escolha do algoritmo de registro tem pouca relevância para o

\footnotetext{
${ }^{2}$ Note que distorções gaussianas não são consideradas em [6].

${ }^{3} \mathrm{O}$ valor ótimo para o passo de convergência apenas poderia ser determinado através do conhecimento das matrizes $\mathbf{M}_{1}(t)$ e $\mathbf{M}_{2}(t)$, o que não é viável em aplicações práticas.
} 


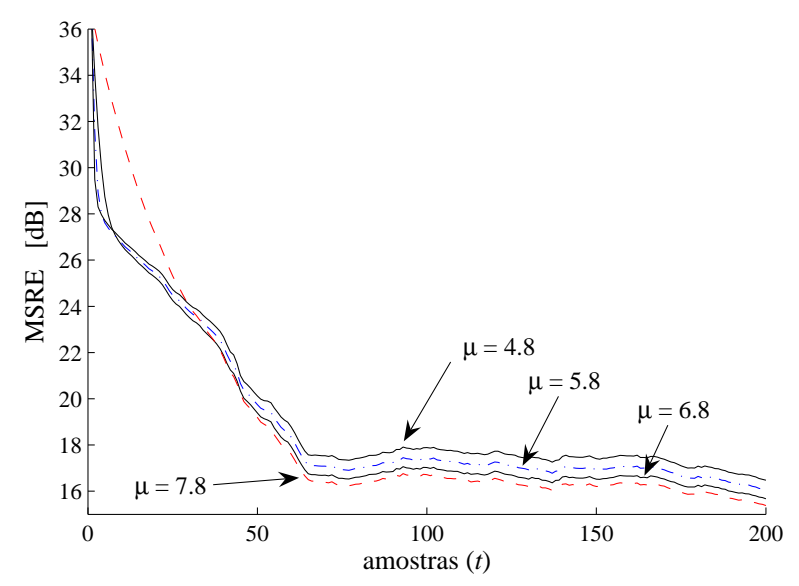

Fig. 2. MSRE considerando blurring gaussiano.

objetivo deste exemplo. Um algoritmo de registro com melhor (pior) desempenho iria apenas atenuar (acentuar) os efeitos do erro de estimação de movimento observados. Interpolações bicúbicas das imagens de baixa-resolução foram utilizadas como sinais de entrada para os algoritmos de registro. O ruído aditivo foi assumido $\operatorname{WGN}\left[0, \sigma_{e}^{2}(t)\right]$, em que $\sigma_{e}^{2}(t)=10$.

Na Figura 3 é mostrada a evolução temporal do MSRE considerando a escolha proposta para o passo de adaptação $(\mu=6,8)$ e diferentes outros passos em torno dessa escolha. Para todos os valores de $\mu$ avaliados, a escolha proposta leva ao mínimo MSRE. A velocidade de convergência é razoavelmente próxima à velocidade alcançada pelo uso de outros passos (mais rápidos), tais como $\mu=5,8$ e $\mu=3$, com uma perda de desempenho que provavelmente é aceitável para a maioria das aplicações práticas.

\section{Exemplo 3: Seqüência de imagens real}

O objetivo deste exemplo é verificar o desempenho do algoritmo LMS-SRR quando aplicado a seqüências de imagens reais, usando a metodologia de projeto proposta. Nesses casos, têm-se presentes tanto outliers resultantes de inovações entre as imagens quanto resultantes do erro de registro.

A seqüência de imagens utilizada neste exemplo, $\mathrm{Hand}^{4}$, inclui movimentos que são mais complexos do que os modelados pelo movimento translacional e global, considerado nos exemplos anteriores. Os quadros originais dessa sequiência foram redimensionados para $128 \times 128$ pixels, via interpolação bicúbica. As imagens de baixa resolução são assumidas como tendo $64 \times 64$ pixels.

O algoritmo de registro utilizado é o proposto em [14]. $\mathrm{O}$ ruído aditivo é assumido $\mathrm{WGN}\left[0, \sigma_{e}^{2}(t)\right], \sigma_{e}^{2}(t)=10$. As distorções ópticas (blurring) e a subamostragem são implementadas exatamente como no Exemplo 1.

Na Figura 4 é mostrada a evolução do erro quadrático médio de reconstrução (considerando média espacial) para diferentes passos de adaptação. Por se tratar de uma única realização,

\footnotetext{
${ }^{4}$ Base de dados do Vision and Autonomous Systems Center; http://vasc.ri.cmu.edu/idb/html/motion/hand/index.html (acessado em 12 de março de 2007)
}

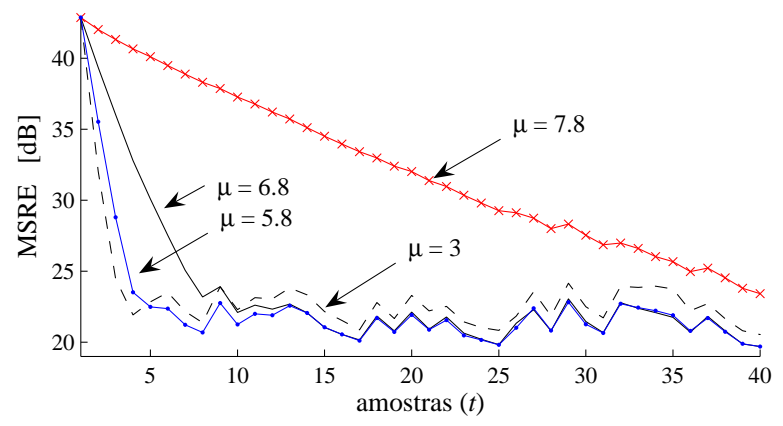

(a)

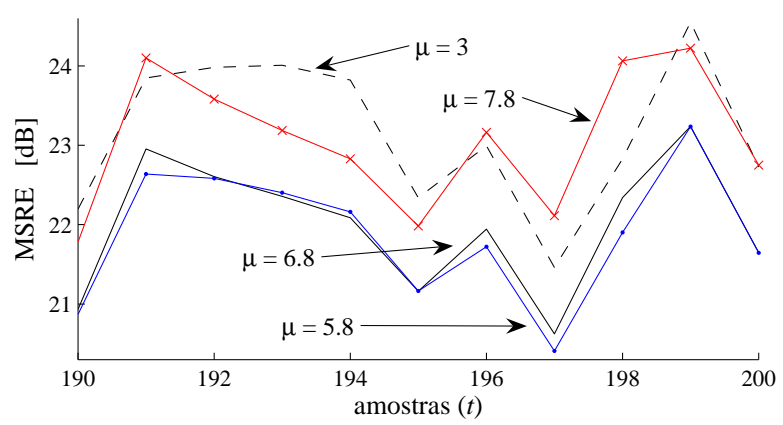

(b)

Fig. 3. MSRE do LMS-SRR considerando erros de registro: (a) zoom do regime transitório; (b) zoom do regime permanente.

as curvas de erro não são tão suaves quanto nos exemplos anteriores, cruzando-se por diversas vezes. Entretanto, cabe destacar que este gráfico não tem a intenção de verificar a validade da análise estatística apresentada. Destina-se apenas a identificar que, mais uma vez, o uso do passo proposto $\mu=\mu_{\mathrm{o}}$ leva a um desempenho próximo do ótimo.

Resultados de reconstrução do $200^{\circ}$ quadro da seqüência, considerando diferentes passos de convergência, são mostrados na Figura 5. Para fins de comparação da qualidade obtida, também é mostrado o resultado da reconstrução por interpolação bicúbica. Pode ser verificado que o passo proposto $\mu=6,8$ leva, de fato, a um resultado perceptual próximo do ótimo, como previsto pelos resultados ilustrados na Figura 4. Nas figuras 5(a) e (b) são mostradas a imagem original e a interpolação bicúbica da imagem observada, respectivamente. A imagem na Figura 5(c) $(\mu=1,0)$ é excessivamente alisada. Nas figuras 5(d) $(\mu=5,8)$ e $5(\mathrm{e})(\mu=$ $6,8)$ as imagens apresentam qualidades bastante similares. Por fim, na Figura 5(f) $(\mu=7,8)$ a imagem é visivelmente mais ruidosa.

\section{CONCLUSÕES}

Neste trabalho foi apresentada uma análise estatística do comportamento do algoritmo LMS aplicado à reconstrução de vídeo com super-resolução na presença de outliers. Baseado nessa análise, foram propostas diretrizes de projeto para a escolha dos parâmetros (passo de convergência e número de iterações do algoritmo por amostra temporal), visando reduzir a influência dos outliers causados pelas inovações entre quadros (movimentos na cena) e pelos erros de registro. Situações 


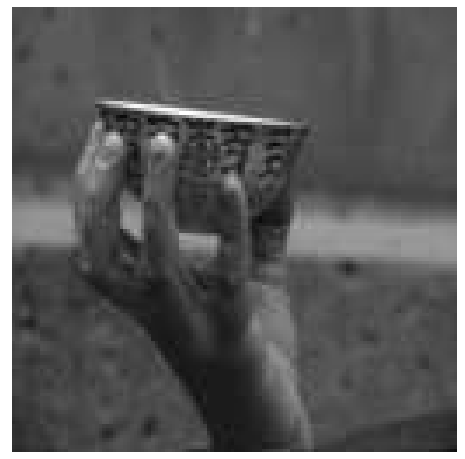

(a)

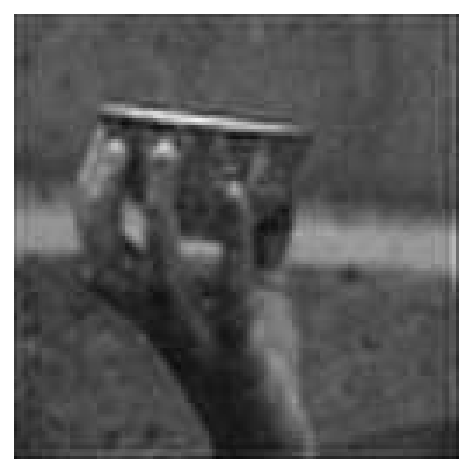

(d)

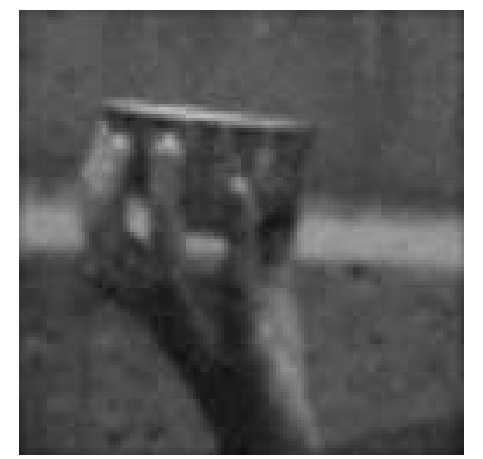

(b)

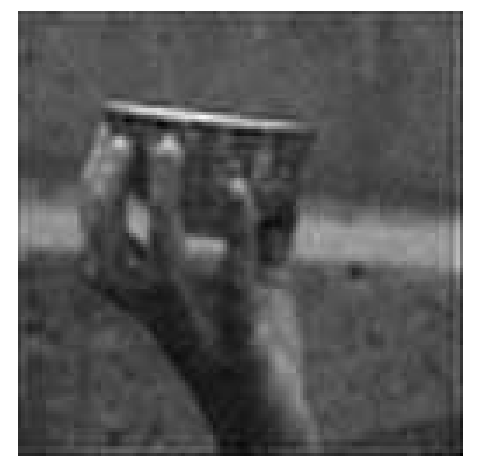

(e)

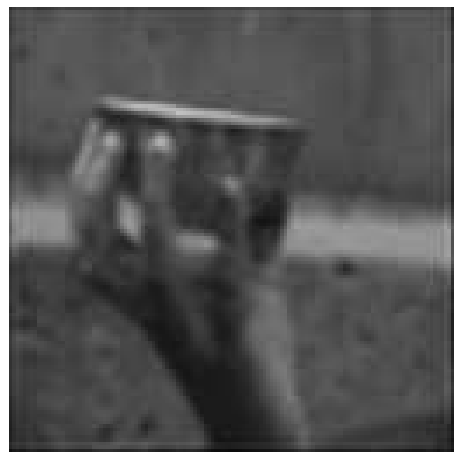

(c)

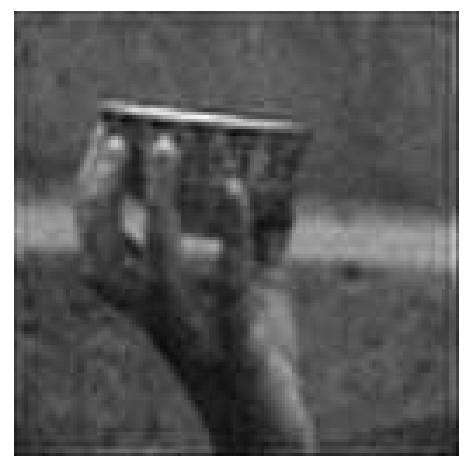

(f)

Fig. 5. Exemplos de resultados para a sequiência Hand: $200^{\circ}$ quadro, (a) imagem original; (b) interpolação bicúbica; (c) $\mu=1$; (d) $\mu=5,8$; (e) $\mu=6,8$; (f) $\mu=7,8$.

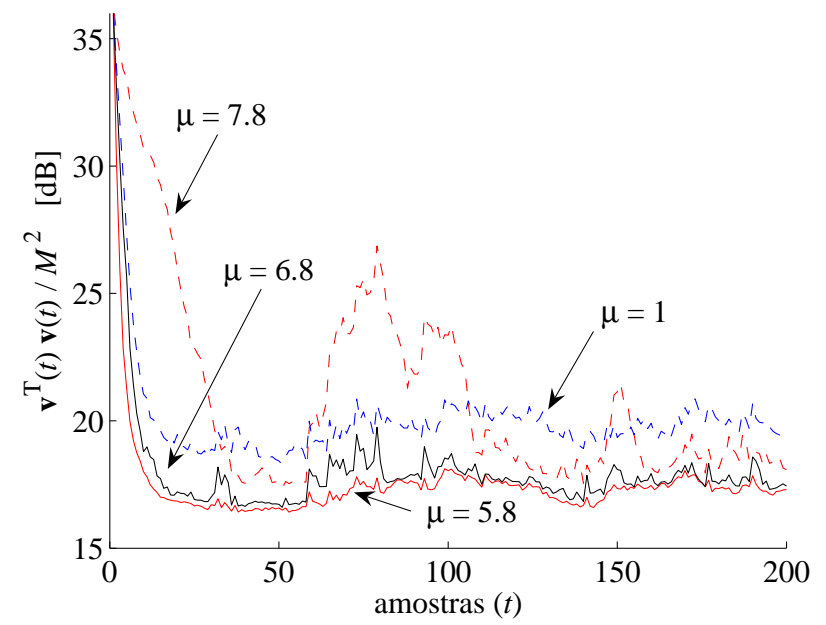

Fig. 4. Erro quadrático médio (amostral) de reconstrução do LMS-SRR, considerando a seqüência Hand.

reais de aplicação foram consideradas. Foi mostrado que, diferente do comportamento do algoritmo LMS tradicional, valores menores do passo de adaptação não levam necessariamente a menores erros quadráticos médios de reconstrução em regime permanente.

\section{REFERÊNCIAS}

[1] S. Park, M. Park, and M. Kang, "Super-resolution image reconstruction: A technical overview," IEEE Signal Process. Magazine, vol. 20, no. 3, pp. 21-36, May 2003.

[2] S. Farsiu, D. Robinson, M. Elad, and P. Milanfar, "Advances and challenges in super-resolution," Int'l Journal of Imaging Syst. and Technology, vol. 14, no. 2, pp. 47-57, Aug. 2004.

[3] A. Zoomet, A. Rav-Acha, and S. Peleg, "Robust super-resolution," IEEE CVPR, vol. 1, pp. 645-650, Dec. 2001.

[4] S. Farsiu, D. Robinson, M. Elad, and P. Milanfar, "Fast and robust multiframe super resolution," IEEE Trans. Image Process., vol. 13, no. 10, pp. 1327-1344, Oct. 2004.

[5] M. Elad and A. Feuer, "Superresolution reconstruction of an image sequence," IEEE Trans. PAMI, vol. 21, no. 9, pp. 817-834, Sept. 1999.

[6] G. Costa and J. Bermudez, "On the design of the LMS algorithm for robustness to outliers in super-resolution video reconstruction," IEEE ICIP, pp. $1-4$, Oct 2006.

[7] G. H. Costa and J. C. M. Bermudez, "Statistical analysis of the LMS algorithm applied to super-resolution image reconstruction," IEEE Trans. Signal Process., vol. 55, no. 5, pp. 1-12, May 2007.

[8] Z. Wang and F. Qi, "Super-resolution video restoration with model uncertainties," IEEE ICIP, vol. 2, pp. 853-856, Sept. 2002.

[9] M. Ng and N. Bose, "Analysis of displacement errors in high-resolution image reconstruction with multisensors," IEEE Trans. Circuits and Syst., vol. 49, no. 6, pp. 806-813, June 2002.

[10] J. Modersitzki, Numerical Methods for Image Registration, 1st ed. Oxford University Press, 2004.

[11] C. Meyer, Matrix Analysis and Applied Linear Algebra. Siam, 2000.

[12] O. Macchi, Adaptive Processing:The Least Mean Squares Approach with Applications in Transmission. Wiley, 1995.

[13] R. Mester and M. Hotter, "Robust displacement vector estimation including a statistical error analysis," IEE Int'l Conf. Image Process. and its Applications, pp. 168-172, July 1995.

[14] B. K. P. Horn and B. G. Schunck, "Determining optical flow," Artificial Intelligence, vol. 17, pp. 185-203, 1981. 